\title{
Hybrid Simulation Approach on MEMS Piezoresistive Microcantilever Sensor for Biosensing Applications
}

\author{
ROSMINAZUIN AB. RAHIM ${ }^{1, a}$, BADARIAH BAIS ${ }^{2, b}$ and BURHANUDDIN \\ YEOP MAJLIS ${ }^{3, C}$ \\ 1,2,3 Institute of Microengineering and Nanoelectronic (IMEN), \\ Universiti Kebangsaan Malaysia \\ 43600, Bangi, Selangor, Malaysia \\ arosminazuin@gmail.com, ${ }^{\mathrm{b}}$ badariah@vlsi.eng.ukm.my, ${ }^{\mathrm{c} b u r h a n @ v l s i . e n g . u k m . m y ~}$
}

Keywords: Biosensor, piezoresistor, cantilever, MEMS

\begin{abstract}
This paper uses a hybrid simulation approach in CoventorWare design environment which combines finite element analysis and circuit simulation modeling to obtain the optimal performance of piezoresistive microcantilever sensor. A $250 \mu \mathrm{m}$ x $100 \mu \mathrm{m}$ x $1 \mu \mathrm{m} \mathrm{SiO}{ }_{2}$ cantilever integrated with $0.2 \mu \mathrm{m}$ thick Si piezoresistor were used in this study. A finite element analysis on piezoresistive microcantilever sensor was conducted in CoventorWare Analyzer environment which incorporates MemMech and MemPZR modules. The sensor sensitivity was obtained by measuring resistivity changes in piezoresistive material in response to surface stress changes of microcantilever. The simulation results were later integrated with system-level simulation solver called Architect to enable the optimization of the sensor circuit output. It involves a hybrid approach which uniquely combined FEM analysis and piezoresistive modeling using circuit simulation environment which results in optimal performance of MEMS piezoresistive microcantilever sensor.
\end{abstract}

\section{Introduction}

A MEMS microcantilever sensor integrated with piezoresistive read out is becoming popular in measuring the surface stress change induced from biochemical reaction since it performs well in liquid environments despite being simple and cheap [1]. However, a low resolution in the read out system limits its sensing capability as the magnitude of the forces or stresses involved in the system is very small. Hence, many previous related researches converged on the optimization of the sensor performance by analyzing the cantilever geometrical dimension and property [2,3]. Conventionally, studies on the MEMS device involved Finite Element Method (FEM) and Boundary Element Method (BEM) analyses, where individual components were analyzed to produce accurate results [4-7]. However, these methods have tradeoffs in time, skill level requirements, limitations on the scope of the design problem that can be analyzed, and restrictions on the types of simulations that can be performed.

System-level simulation technique from Architect solver offers alternate approach for MEMS analysis. This technique performs simulations based on the behavior of a device as expressed by reduced-order equations. It simulates the overall behavior of a complete model instead of the interactive behavior of many finite elements that comprise the model which reduces the number of computations and resulting in much faster simulation runs.

This research uses a hybrid simulation approach to obtain the optimal performance of $\mathrm{SiO}_{2}$-based piezoresistive microcantilever sensor by combining FEM analysis and piezoresistor modeling using system-level simulation environment. The integration of the sensor output circuit with Wheatstone Bridge circuit yields the sensor response in terms of output voltage as a function of applied pressure. 


\section{Theory}

The piezoresistor, PZR network has the Wheatstone bridge configuration to measure small changes in resistance as illustrated in Fig. 1. The relationship between the voltage and the resistance is given as

$$
\frac{V_{o}}{V_{s}}=\frac{R_{1} R_{3}-R_{2} R_{4}}{\left(R_{1}+R_{2}\right)\left(R_{3}+R_{4}\right)} .
$$

In Eq. $1, V_{o}$ is the voltage at the PZR network output and $V_{s}$ is the input voltage. $R_{1}$ is represented by the Straight Piezoresistor component while $R_{2}, R_{3}$ and $R_{4}$ are fixed resistors with values of $1 \mathrm{k} \Omega$.

$V_{o}$ is then connected as an input in a difference amplifier circuit based on an OpAmp, Ideal 3-pin and a resistor network. The output is defined in Eq. 2 as

$$
V_{\text {out }}=V_{o} \cdot \frac{R_{2}}{R_{1}}
$$

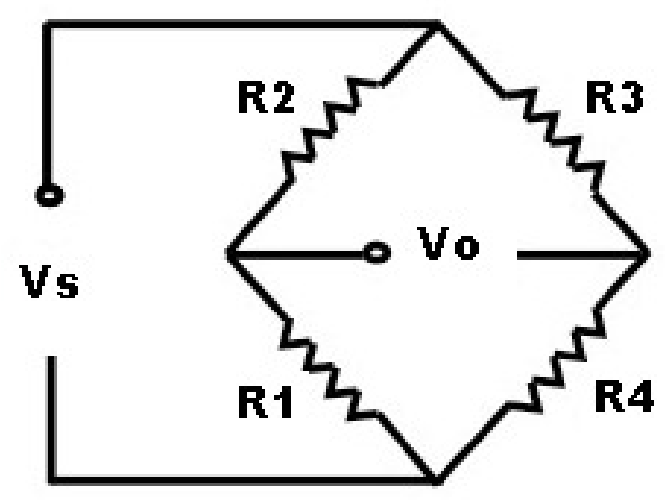

Fig. 1 Wheatstone bridge circuit.

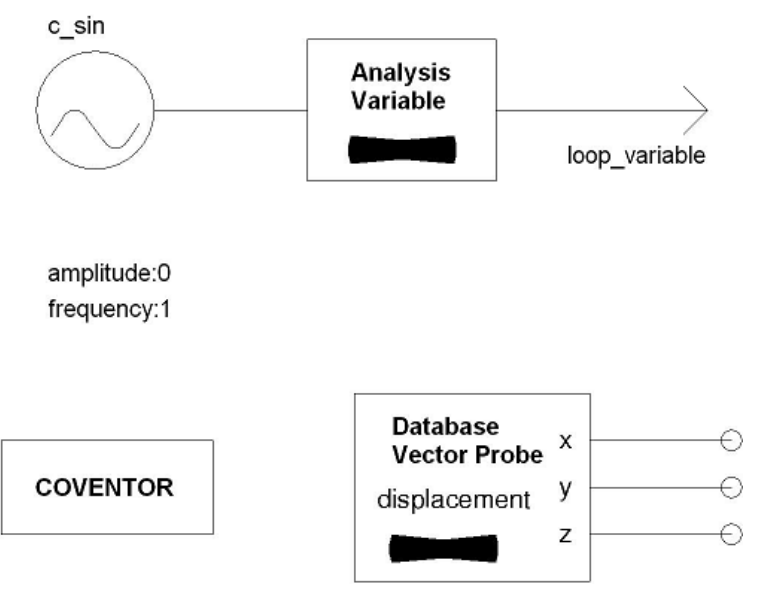

Fig. 2 Circuit configuration in Architect solver.

\section{Design Simulation \& Analysis}

The first stage of the simulation work involved the analysis of a rectangular $\mathrm{SiO}_{2}$ cantilever beam only (i.e. without piezoresistor) using both mechanical FEM and circuit simulation solvers. In the second stage, the analysis of integrated piezoresistor on the cantilever beam was conducted using Architect by extracting data from the Analyzer database.

Fig. 3 shows the behavior of cantilever beam deformation obtained from Analyzer and Architect solvers, respectively. Using the Analyzer solver, a parametric study was conducted by observing the cantilever beam deformation under a series of different applied pressures. From Fig. 3, a maximum beam displacement of $8.259 \times 10^{-4} \mu \mathrm{m}$ is observed at maximum load of $0.1 \mu \mathrm{N}$. The FEM results can also be visualized by accessing the CoventorWare database using Architect environment as shown in Fig. 2. From the simulation, it was found that the results obtained by the Analyzer solver agree with the one obtained by the Architect. This shows that Architect is a reliable tool in measuring the behavior of cantilever beam deformation.

The piezoresistor design and optimization was conducted by simulating sensor output circuit after specifying the piezoresistor layer properties in Analyzer. The piezoresistor parameters were then defined in Architect by considering that all database access models in the schematic use the same FEM database results to ensure correct placement of piezoresistor in relation to the cantilever beam developed in the initial stage of simulation. From the schematic shown in Fig. 4, it can be 
seen that the piezoresistor is then connected to the Wheatstone bridge circuit to measure the sensor circuit output voltage.

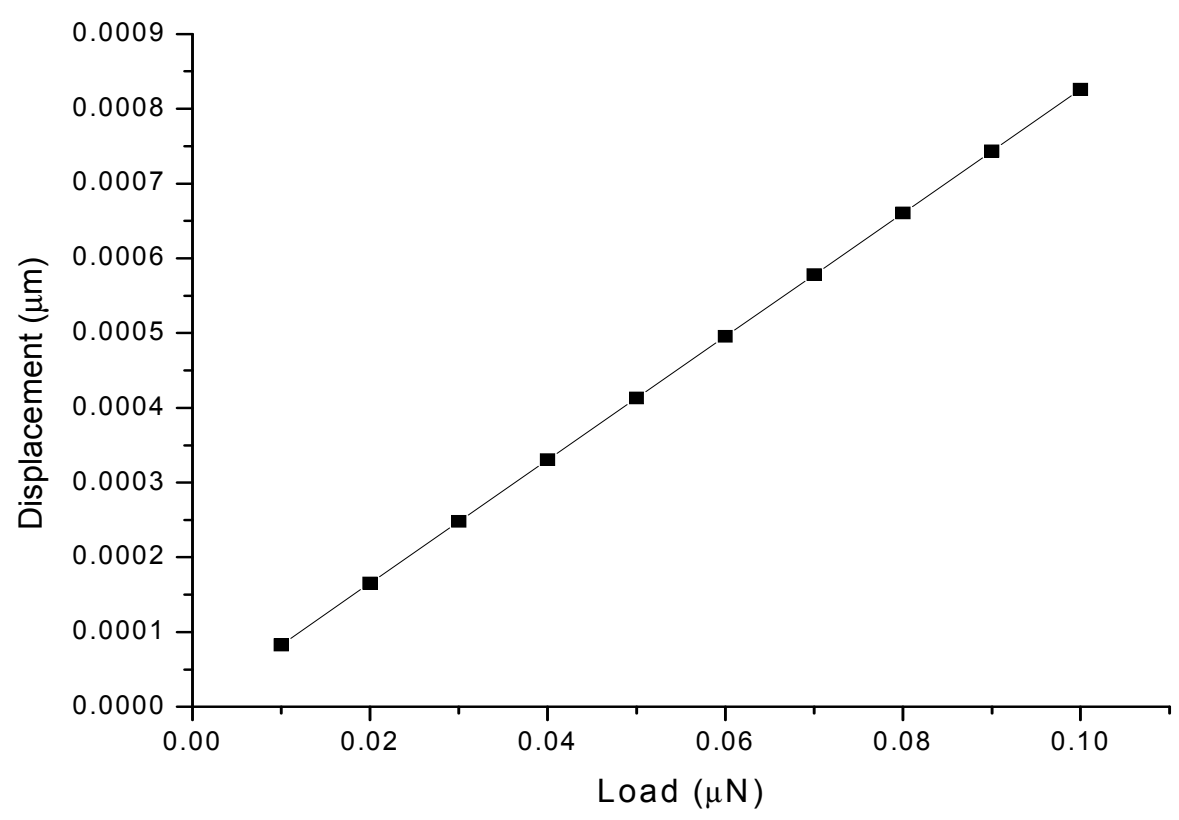

Fig. 3 Cantilever displacement as a function of applied load from Analyzer solver.
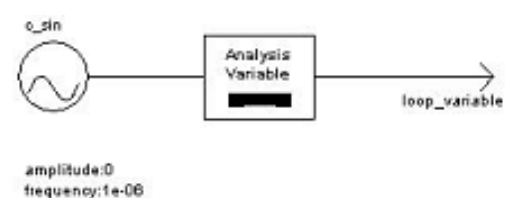

frequency:19.06

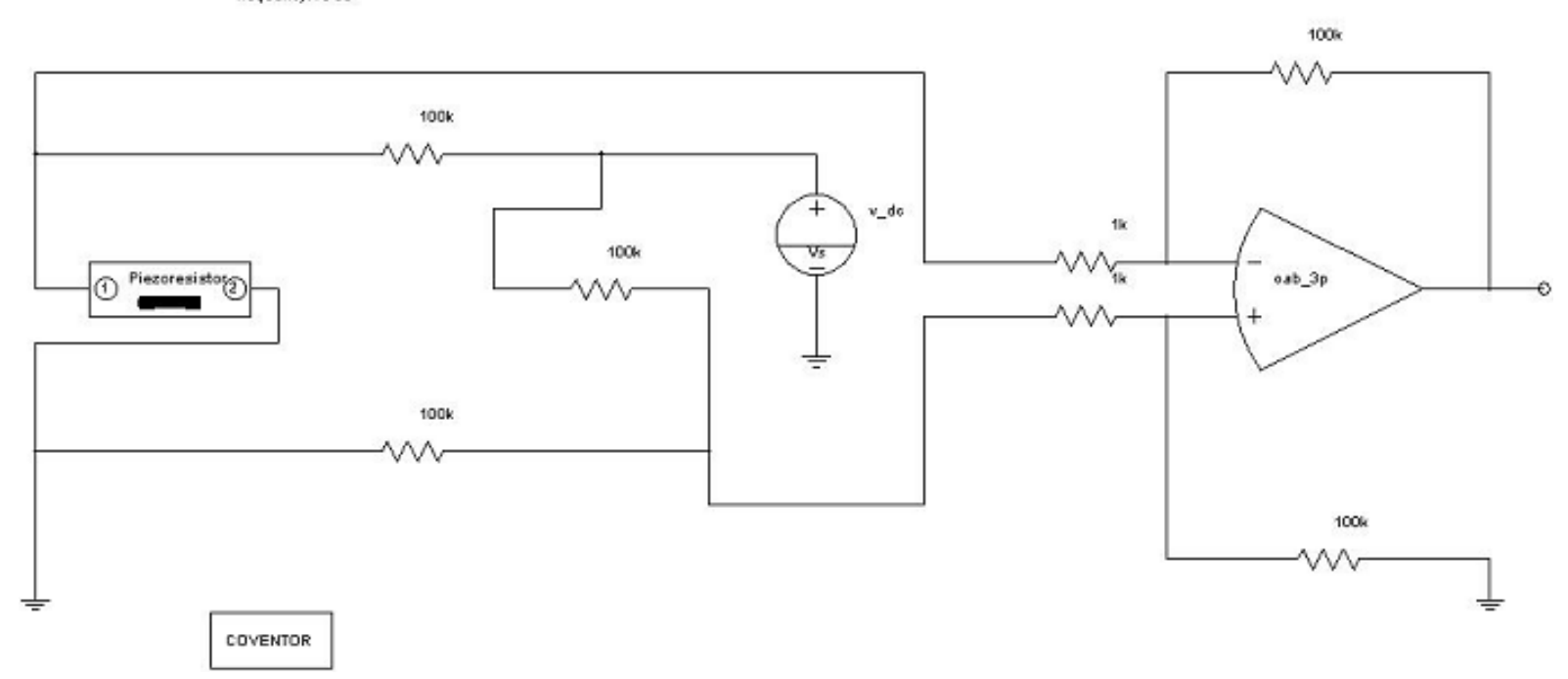

Fig. 4 Sensor output circuit extracted from Sabersketch in Architect solver.

Using DC transfer analysis, simulation on the sensor output signal was conducted. The simulation was performed under the same pressure range defined in Analyzer previously. Fig. 5 shows the sensor output generated from CosmosScope of the Architect solver. From Fig. 5, it is shown that the sensor output voltage is linearly proportional to the applied pressure. Since the applied pressure represents the stress in sensor reaction, it is expected that as the applied pressure increases, the cantilever beam displacement also increases resulting in the increment of sensor 
output voltage. Note that the sensor response yields inverted results as shown in Fig. 5 since inverting opAmp was used in the circuit configuration.

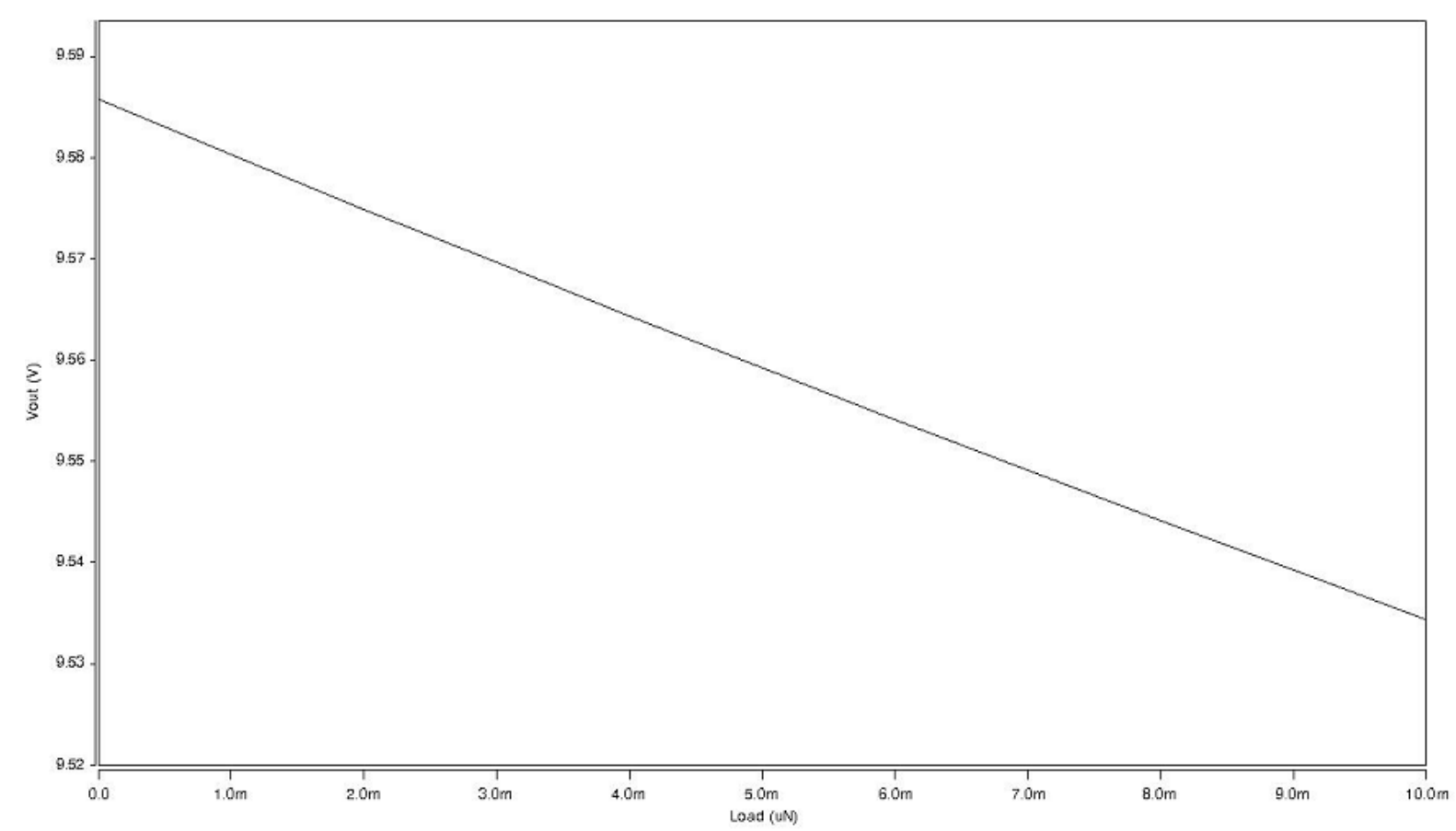

Fig. 5 Sensor output voltage as a function of applied pressure generated from CosmoScope in Architect solver.

\section{Conclusion}

The integration of both FEM and circuit simulation solvers in Coventorware enables the optimization of MEMS piezoresistive cantilever-based sensor with faster simulation time. Simulation work on the complex interactive behavior of finite elements in Analyzer show similar results with simulation on overall behavior of the piezoresistive microcantilever-based sensor model in Architect at a reduced computation time.

\section{References}

[1] M. Tortonese, H. Yamada, R.C. Barrett and C.F. Quate: Proc. Transducers'91 (1991), p. 448.

[2] Z.Y. Wang, R.F. Yue, R.X. Zhang and L.T. Liu: Sensors and Actuators A Vol. 120 (2005), p. 325.

[3] S.M. Yang and T.I. Lin: Sens. Actuat. B Vol. 120 (2007), p. 736.

[4] P.A. Rasmussen, O. Hansen and A. Boisen: Applied Physics Letters Vol. 86 (2005), p. 203.

[5] S. Kassenge, J.M.M.R.W., J. Zoval, E. Mather, K. Sarkar, D. Hodko and S. Maity: presented at the 2002 SPIE Conf on Smart Structure and Materials, San Diego, CA, (2002).

[6] M.A. Bhatti, L.C. Xi, L.Y. Zhong and A.N. Abdalla: presented at the 2007 Second IEEE Conference on Industrial Electronics and Applications, Harbin, China, (2007).

[7] Rahim, R.A.; Bais, B.; Majlis, B.Y.: IEEE ICSE 2008 (2008), p.211. 